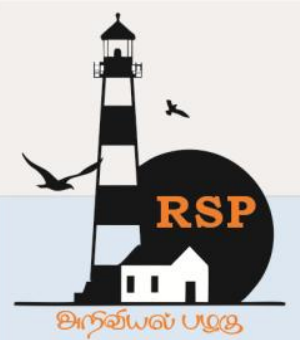

INTERNATIONAL RESEARCH JOURNAL ON ADVANCED SCIENCE HUB

\title{
Electrical properties of polyaniline/Cadmium Oxide/ZnO Nanocomposites Thin Films
}

\author{
Jakeer Husain ${ }^{1}$, Rehana Anjum ${ }^{2}$, Shivaraj. $G^{3}$, Gavisiddayya.Mathad ${ }^{4}$, Deepa Pathar ${ }^{5}$, Jaisheel Sagar ${ }^{6}$, \\ Bushara Anjum ${ }^{7}$ \\ ${ }^{1}$ Assistant Professor Dept of Engg Physics Faculty of Engineering and Technology KBNU Gulbarga, \\ karnataka \\ ${ }^{2}$ Professor S\&H and HOD Chemistry Lords Institute of Engineering and Technology, Hydrabad \\ ${ }^{3}$ Assistant Professor Dept of Physics Govt First Grade college Yelburga, Koppal, Karnataka \\ ${ }^{4}$ Lecturer Dept of Physics Govt First Grade college Yelburga, Koppal, Karnataka \\ ${ }^{5}$ Lecturer Dept of Physics Govt First Grade college Yelburga, Koppal, Karnataka \\ ${ }^{6}$ Assistant Professor Mechanical Engg Department, FIT, Chevella, JNTUH, India, \\ ${ }^{7}$ Nagambika BEd College Gulbarga \\ jakeerhusain21@gmail.com ${ }^{1}$
}

\begin{abstract}
Polyaniline nanocomposite films were prepared with various weight percentages by in situ polymerization method using aniline, ammonium per sulfate, and cdo as starting materials. The formations of pani and pani/cdo/ZnO composites with regards to the structural and microstructural properties of the materials were investigated by SEM techniques. The DC conductivity of Pure Pani and its composites have been measured in the temperature range from $30^{\circ} \mathrm{C}$ to $160^{\circ} \mathrm{C}$.
\end{abstract}

Keywords: ZnO,CdO,SEM,PANI,Composite .

\section{Introduction}

Among conducting polymers, polyaniline family has attracted much attention of scientists worldwide because of their ease of synthesis, unique conduction mechanism, high environmental stability in the presence of oxygen and water, low cost, light weight and good sensing capability[13]. They also exhibit highly reversible redox behavior, which is very important for many applications. Extensive literature review suggests that the considerable efforts have been made by researchers all over the world in improving the conductivity of polyaniline by various doping techniques, but little is known about the dielectric properties of conducting polymers associated with conduction mechanism [4] .On doping these conjugated polymers show very high conductivity similar to metals. Therefore sometimes they are also called synthetic metals. They combine the electrical properties of metals with the advantage of polymers such as smaller weight, greater workability, resistance to corrosion and lower cost [5-7].

\section{Experimental procedures:}

2.1 Chemical synthesis of polyaniline.

The synthesis was based on mixing aqueous solution of aniline hydrochloride and ammonium persulphate at room temperature, followed by the separation of PANI hydrochloride precipitate by filtration and drying. Aniline hydrochloride (equi molar volume of aniline and hydrochloride acid) was dissolved in distilled water in a volumetric flaskto $100 \mathrm{ml}$ of solution. Ammoniumpersulphate $(0.25 \mathrm{M})$ was dissolved in water and also to $100 \mathrm{ml}$ of solution. Both solutions were kept for 1 hour at room temperature, then mixed in a beaker, stirred 
with a mechanical stirrer, and left at rest to polymerize. Next day, the PANI precipitate was collected on a filter, washed with 0.2 M HCL, and similarly with acetone. Polyaniline hydrochloride powder was dried in air and then in vacuum at $60^{\circ} \mathrm{C}$ for 24 hours. Polyaniline prepared under these reaction and processing conditions are further referred to as "standard" samples.

\section{2 preparations of polyaniline/ cdo/zno composites}

Synthesis of the PANI-Cdo/ZnO composites was carried out by in-situ polymerization method. Aniline $(0.1 \mathrm{M})$ was mixed in $1 \mathrm{M} \mathrm{HCl}$ and stirred for $15 \mathrm{~min}$ to form aniline hydrochloride. $\mathrm{Cdo} / \mathrm{ZnO}$ particles were added in the mass fraction to the above solution with vigorous stirring in order to keep the $\mathrm{Cdo} / \mathrm{ZnO}$ homogeneously suspended in the solution. To this solution, $0.1 \mathrm{M}$ of ammonium persulphate, which acts as an oxidizer was slowly added drop-wise with continuous stirring at $5^{\circ} \mathrm{C}$ for $4 \mathrm{~h}$ to completely polymerize. The precipitate was filtered, washed with deionised water, Acetone, and finally dried in an oven for $24 \mathrm{~h}$ to achieve a constant mass. In these ways, PANI- $\mathrm{Cdo} / \mathrm{ZnO}$ composites containing various weight percentage of $\mathrm{Cdo} / \mathrm{ZnO}$ $(10 \%, 20 \%, 30 \%, 40 \%$, and $50 \%)$ in PANI were synthesized.

\section{Results and discussions}

\subsection{Scanning Electron Microscope}

Figure 1 (a) shows that Scanning Electronic Micrograph (SEM) image of pure PANI, which has highly agglomerated granular in shape. The grains are well interconnected with each other which indicate that they have enough binding energy to combine with neighbour grains or molecules. The sem image of pani-cdo/ZnO composite $(50 \%)$ is shown in figure $1 \mathrm{~b}$ ). It can be seen from the image that the particle having spherical and porous structure.

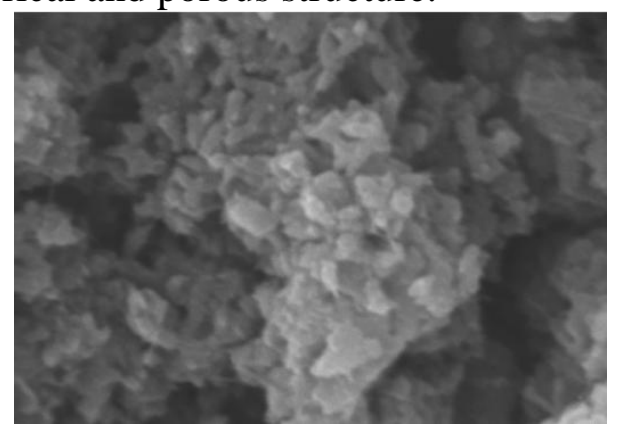

a) PANI

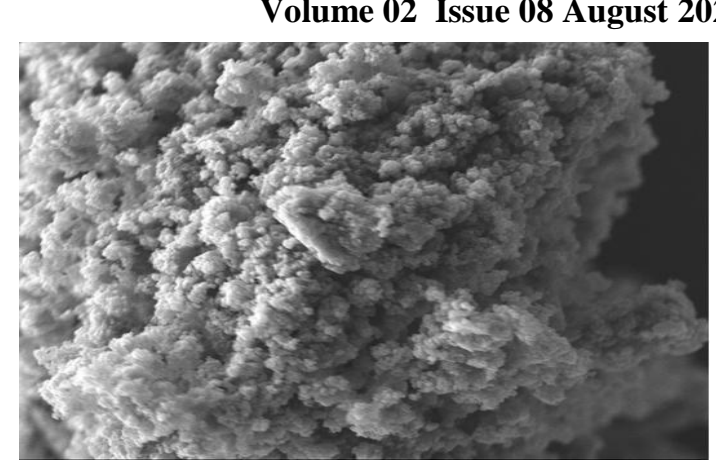

b) PANI/ $\mathrm{CdO} / \mathrm{ZnO}$ Composite

\section{Figure 1 (a\&b) shows that Scanning Electronic Micrograph (SEM) image of pure PANI and CdO/ZnO Composite \\ 4 DC Conductivity}

The variation of conductivity with temperature for pani-cdo/ZnO composites are shown in figure 4. The increase in conductivity for the composites containing $\mathrm{Cdo} / \mathrm{ZnO}$ particles may be due to the formation of more number of polarons in the widen band gap. Hence for the particular amount of filler material, the surface of pani would be modified to have high conductivity and have much active surface. The change in the conductivity of the composites indicates a change in the doping state of the polymer. Though the composite having $50 \%$ of $\mathrm{cdo} / \mathrm{ZnO}$ shows the higher conductivity than pure pani and other composites but the formation of 3-d conduction network is not likely and thus such enhancement of dc conductivity values can be attributed to the uncoiling of polymeric chains due to strong interfacial interaction between $\mathrm{cdo} / \mathrm{ZnO}$ crystallites with pani caused by their composition.

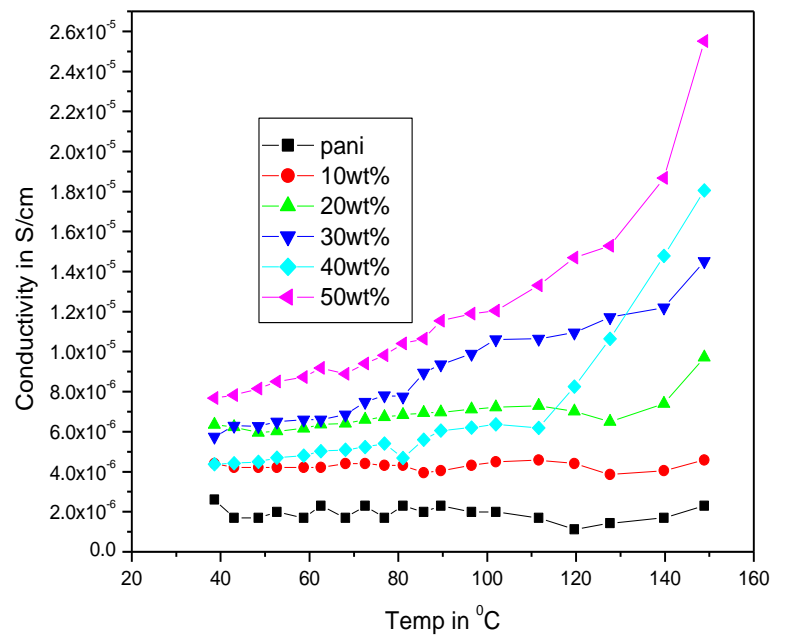

Fig.2. Shows the DC conductivity of PANI and $\mathrm{CdO} / \mathrm{ZnO}$ nanocomposite thin films 


\section{Conclusion}

In this study pani/cdo/ZnO nanocomposite were successfully synthesized by in-situ polymerization method in the presence of cdo nanoparticles.the results of SEM show the formation of the composite and indicate an interaction between pani and cdo nanoparticles.the. The electrical properties of pani/ cdo/ $\mathrm{ZnO}$ nanocomposite are increases with increasing in frequency it's because of interaction between metal oxide and pani. Hence this composite is a promising material for potential applications.

\section{References}

[1].Synthesis, characterization and electrical properties of polyaniline/batio3 composites. Ferroelectrics, 325,2005, 111116.

[2].R. Sinha, outline s of polymer technology, new delhi:prentice hall of india private limited, (2002).

[3]. Chemical synthesis characterization and dc conductivity of polypyrrole $-\gamma-\mathrm{fe} 2 \mathrm{o} 3$ composites. J. Appl. Poly. Sci.103, 2007, 2797-2805.

[4].K.Yoshino, M.Ozaki and R.Sugimoto, Jpn.J.Appl.Phys.Lett. ,1985, 24, 373.

[5].J.C.Chiang and A.G.Mac Diarmid, Synth. Met, 1986,13, 193.

[6].Dr.Jakeer Husain et almaterials today proceedings.2016.

[7].JakeerHusainetal2020J.Phys.:Conf.Ser.149 5012008 